Gesnerus 57 (2000) 304-310

\title{
Contents of Vol. 57 (2000)
}

\section{Original Articles}

Halleux, R.: L'histoire des sciences au seuil du troisième millénaire. Des hommes, des idées et des institutions. [The History of Sciences at the Threshold of the Third Millenium.

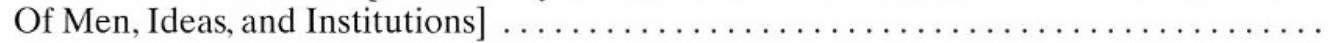

Jacobi, Manfred: Wolfgang Paulis familiärer Hintergrund [Wolfgang Pauli's Family Back-

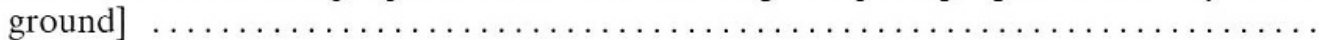

Maehle, Andreas-Holger: Assault and Battery, or Legitimate Treatment? German Legal Debates on the Status of Medical Interventions without Consent, c. 1890-1914 ........

Rieder, Philip: La Maternité de Genève (1874-1907), une nouvelle porte d'entrée dans la vie? [Geneva's Maternity Hospital (1874-1907)] . .

Sauer, Tilman: Hilberts Ruf nach Bern [Hilbert's Call to Bern]

Singy, Patrick: Le pouvoir de la science dans L'Onanisme de Tissot [The Power of Science

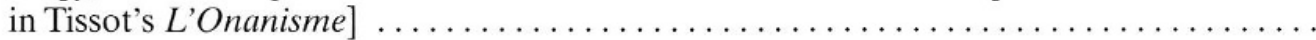

Stuber, Martin: Forschungsreisen im Studierzimmer. Zur Rezeption der Grossen Nordischen Expedition (1733-1743) bei Albrecht von Haller und Samuel Engel [The Reception of the Great Nordic Expedition (1733-1745) by Albrecht von Haller and Samuel Engel] . .

Tavera, Marie: Le rêve naturel: physiologie de l'onirisme au XVIII ${ }^{\mathrm{e}}$ siècle [The Natural Dream: The Physiology of Dreaming in the 18 th Century $] \ldots \ldots \ldots \ldots \ldots \ldots \ldots \ldots$

Weiner, Dora B.: Betrayal! The 1806 English Translation of Pinel's Traité médico-philosophique sur l'aliénation mentale ou la manie.

\section{Short Communications}

Daxecker, Franz: Der Jesuit Athanasius Kircher und sein Organum mathematicum [Athanasius Kircher, SJ, and his Organum mathematicum] ...................

Grigorova, Valentina: Médicaments et thermalisme à Pautalia, Thrace [Drugs and

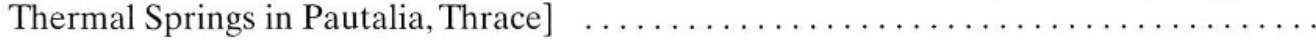

Karbowski, Kazimierz: Au sujet des prénoms de Tissot (1728-1797) [Tissot's Christian Names] 
Book Reviews

Acot, Pascal (ed.): The European origins of scientific ecology (1800-1901) (Junker) . . . 101

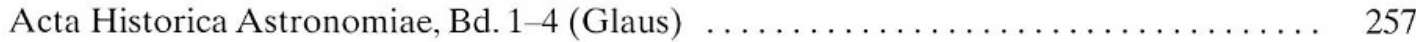

Albou, Philippe: L'image des personnes âgées à travers l'histoire (Hugger) . . . . . . 102

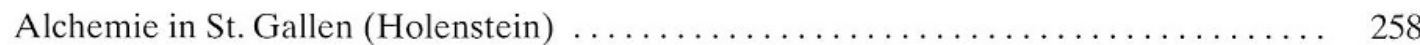

Bachmann, Manuel; Hofmeier, Thomas: Geheimnisse der Alchemie (Gantenbein) . . . . 103

Baldwin, Peter: Contagion and the state in Europe, 1830-1930 (Gutzwiller) . . . . . . . 104

Barona, Josep Lluís; Gómez Font, Xavier: La correspondencia de Carolus Clusius con los científicos españoles (Keller-Schnider) . . . . . . . . . . . . . . . . . . . . . . . .

Bartlett, Peter; Wright, David (eds): Outside the walls of the asylum. The history of care in the community $1750-2000$ (Steinbrecher)

Bénézet, Jean-Pierre: Pharmacie et médicament en Méditerranée occidentale (XIII ${ }^{\mathrm{e}}$ $\mathrm{XVI}^{\mathrm{e}}$ siècles) (Ledermann)

Beretta, Marco; Pogliano, Claudio; Redondi, Pietro (eds): Journals and History of Science (Simon)

Bergdolt, Klaus: Leib und Seele. Eine Kulturgeschichte des gesunden Lebens (Grosche) 261

Die gesammelten Werke der Mathematiker und Physiker der Familie Bernoulli: Die Werke von Jakob Bernoulli, Band 5: Differentialgeometrie (Rummler) 106

Bewersdorff, Jörg: Glück, Logik und Bluff (Ineichen)

Birchler-Argyros, Urs Benno: Quellen zur Spitalgeschichte im Oströmischen Reich (Leven)

Bleker, Johanna (Hrsg.): Der Eintritt der Frauen in die Gelehrtenrepublik (Müller-

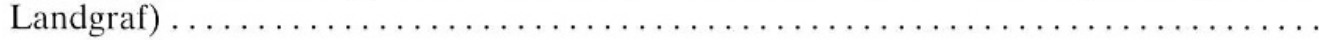

Bödeker, Hans Erich; Reill, Peter Hanns; Schlumbohm, Jürgen (Hrsg.): Wissenschaft als

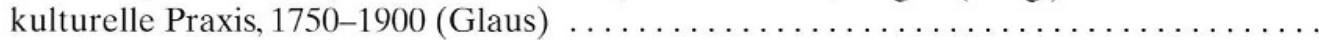

Boi, Luciano; Giacardi, Livia; Tazzioli, Rossana: La découverte de la géométrie non euclidienne sur la pseudosphère. Les lettres d'Eugenio Beltrami à Jules Hoüel (1868-

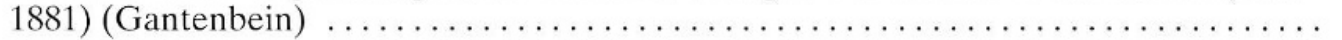

Broman, Thomas H.: The transformation of German academic medicine, 1750-1820

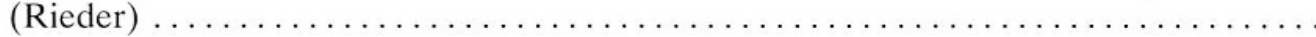


Brosche, P.; Dick, Wolfgang R.; Schwarz, O.; Wielen, R.: The message of the angles - astrometry from 1798 to 1998 (Glaus) . . . . . . . . . . . . . . . . . . . . . . . . .

Carpanetto, Dino: Scienza e arte del guarire. Cultura, formazione universitaria e professioni mediche a Torino tra sei e settecento (Premuda) $\ldots \ldots \ldots \ldots \ldots \ldots \ldots \ldots \ldots$

Cherni, Amor: Epistémologie de la transparence. Sur l'embryologie de A. von Haller

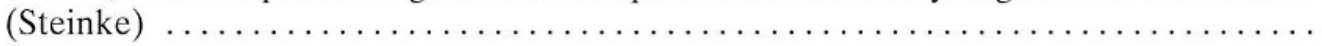

Cimino, Guido; Duchesneau, François (eds): Vitalisms from Haller to Cell Theory

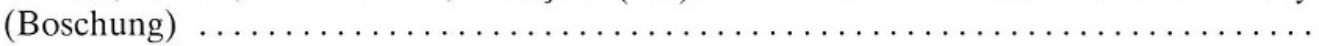

Corsini, Carlo A.; Viazzo, Pier Paolo (eds): The decline of infant and child mortality.

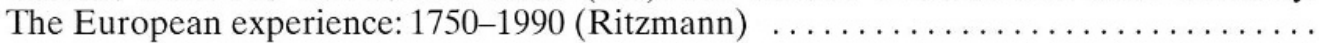

Danón, José (coordinador): La enseñanza de la medicina en la Universidad Española (Keller-Schnider)

Daxecker, Franz; Subaric, Lav (Hrsg.): Christoph Scheiners «Sol ellipticus» (Rieder) ... 285

Deroux, Carl (éd.): Maladie et maladies dans les textes latins antiques et médiévaux

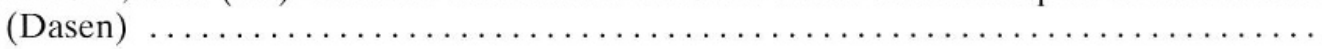

Dick, Wolfgang R.; Hamel, Jürgen (Hrsg.): Beiträge zur Astronomiegeschichte (Glaus) . Dijstelberge, Paul; Noordegraaf, Leo: Plague and print in the Netherlands (Koelbing) ..

Dinges, Martin (Hrsg.): Hausväter, Priester, Kastraten. Zur Konstruktion von Männlichkeit in Spätmittelalter und Früher Neuzeit (Steinbrecher) .....................

Dörner, Klaus (Hrsg.): Ende der Veranstaltung. Anfänge der Chronisch-Kranken-

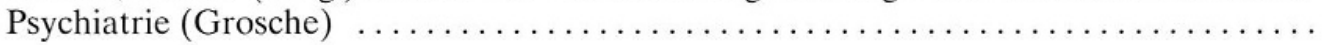

Ebert, Andreas; Weitzel, Hans Karl (Hrsg.): Die Berliner Gesellschaft für Geburtshilfe

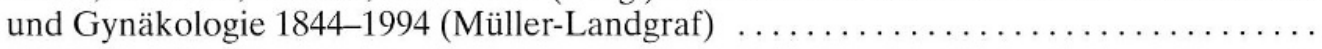

Engstrom, Eric J.; Weber, Matthias M.; Hoff, Paul (eds): Knowledge and power: perspec-

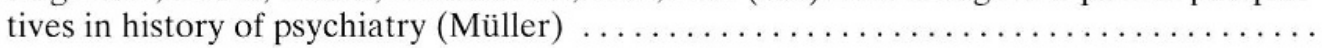

Fischer-Homberger, Esther: Hunger - Herz - Schmerz - Geschlecht. Brüche und Fugen

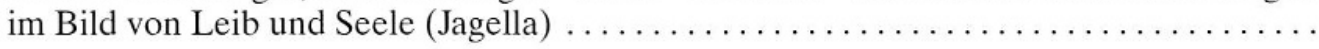

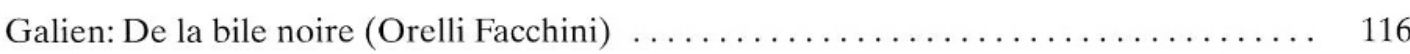

Gayon, Jean: Darwinism's struggle for survival. Heredity and the hypothesis of natural

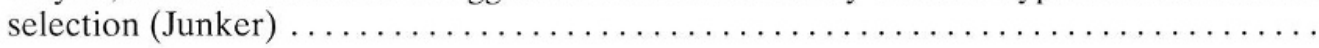

Gijswijt-Hofstra, Marijke; Porter, Roy (eds): Cultures of psychiatry and mental health

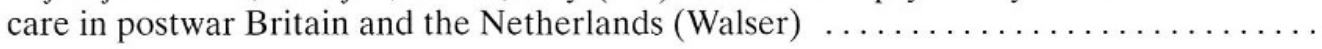

Golgi, Camillo: Sulla fina anatomia degli organi centrali del sistema nervoso (Isler) ....

Gosteli, Leo; Boschung, Urs; Brosche, Peter (Hrsg.): Astronom, Weltbürger, Blasensteinpatient. Franz Xaver Zachs Briefe an Rudolf Abraham von Schiferli 1821-1832 (Glaus)

Gradmann, Christoph; Schlich, Thomas (Hrsg.): Strategien der Kausalität. Konzepte der Krankheitsverursachung im 19. und 20. Jahrhundert (Bröer) $\ldots \ldots \ldots \ldots \ldots \ldots$ 
Grmek, Mirko D.: Le legs de Claude Bernard (Barras) $\ldots \ldots \ldots \ldots \ldots \ldots \ldots \ldots$ 122

Grmek, Mirko D.; Gourevitch, Danielle: Les maladies dans l'art antique (Dasen) ......

Grosser, Hartmut: Historische Gegenstände an der Universitäts-Sternwarte Göttingen

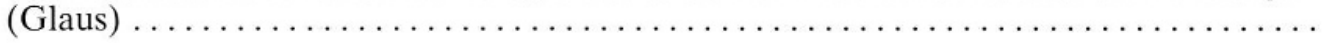

Hänni, Catherine: Im Spannungsfeld zwischen Arzneimittel und Rauschgift (Bickel) .. .

Hahn, Ralf: Gold aus dem Meer. Die Forschungen des Nobelpreisträgers Fritz Haber

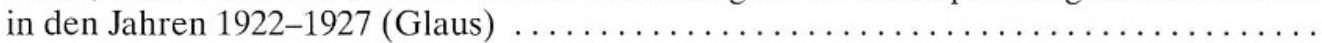

Hamel, Jürgen (Hrsg.): Die astronomischen Forschungen in Kassel unter Wilhelm IV.

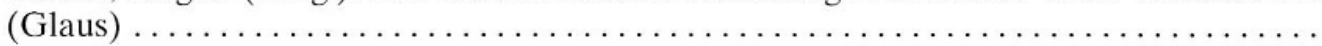

Hars, Florian: Ferdinand Braun (1850-1918). Ein wilhelminischer Physiker (Meyer) ... 257

Heilemann, Hubert Gottfried: Patient Goethe (Grosche)

Heim, Urs F. A.: Leben für Andere. Die Krankenpflege der Diakonissen und Ordens-

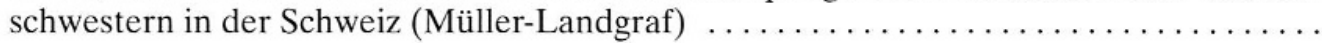

Herbst, K.-D.: Astronomie um 1700. Kommentierte Edition des Briefes von Gottfried Kirch an Olaus Römer vom 25. Oktober 1703 (Glaus) . . . . . . . . . . . . . .

Hörz, Herbert: Brückenschlag zwischen zwei Kulturen. Helmholtz in der Korrespondenz mit Geisteswissenschaftlern und Künstlern (Balsiger) $\ldots \ldots \ldots \ldots \ldots \ldots \ldots \ldots \ldots$

Hummel, Christine: Das Kind und seine Krankheiten in der griechischen Medizin. Von Aretaios bis Johannes Aktuarios (1. bis 14. Jahrhundert) (Perschak; Hoyningen-Süess) .

Jauch, Ursula Pia: Jenseits der Maschine. Philosophie, Ironie und Ästhetik bei Julien

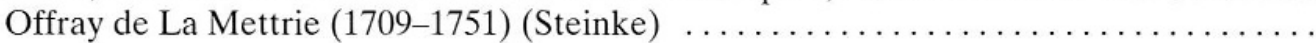

Jones, Peter Murray: Medieval medicine in illuminated manuscripts (Bergdolt) . . . . . .

Keim, Ingeborg M.: Die institutionelle Entwicklung der Kinder- und Jugendpsychiatrie

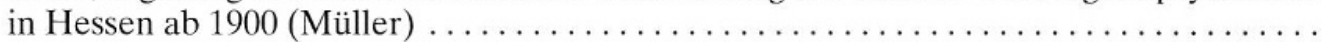

Kirk, Beate: Der Contergan-Fall: eine unvermeidliche Arzneimittelkatastrophe? Zur Geschichte des Arzneistoffs Thalidomid (Ledermann) $\ldots \ldots \ldots \ldots \ldots \ldots \ldots \ldots \ldots$ 274

Klages, Helmut; Lübbig Heinz (Hrsg.): Hermann von Helmholtz, Klassiker an der

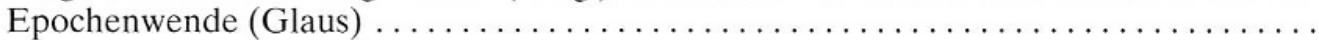

Kohl, Ulrike: Die Kaiser-Wilhelm-Gesellschaft zur Förderung der Wissenschaften im Nationalsozialismus. Quelleninventar (Glaus)

Lauterbach, Irene R.: Christian Wilhelm Hermann Trommsdorff (1811-1884). Zu Leben und Werk eines pharmazeutischen Unternehmers (Ledermann) $\ldots \ldots \ldots \ldots \ldots \ldots$

Lengwiler, Martin: Zwischen Klinik und Kaserne. Die Geschichte der Militärpsychiatrie in Deutschland und der Schweiz $1870-1914$ (Müller) $\ldots \ldots \ldots \ldots \ldots \ldots \ldots \ldots \ldots$

Litsios, Socrates: The tomorrow of malaria (Lindenmann) $\ldots \ldots \ldots \ldots \ldots \ldots \ldots$

Locqueneux, Robert: Préhistoire \& histoire de la thermodynamique classique (Meyer) . 
Lydon, Paul: A catalogue of records retained by hospices and related organisations in the

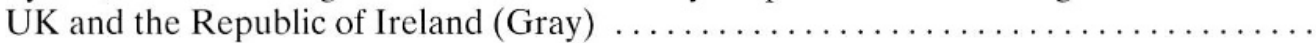

Maehle, Andreas-Holger: Drugs on trial: experimental pharmacology and therapeutic

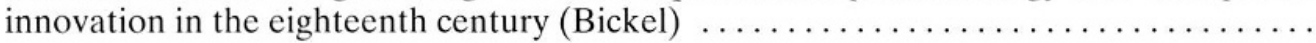

Matussek, Peter (Hrsg.): Goethe und die Verzeitlichung der Natur (Hagner) . . . . . . . .

Mayr, Ernst: Das ist Biologie. Die Wissenschaft des Lebens (Junker)

Meffert, Ekkehard: Carl Gustav Carus. Arzt, Künstler, Goetheanist (Grosche)

Mocek, Reinhard: Die werdende Form. Eine Geschichte der Kausalen Morphologie (Grosche)

Montiel, Luis; Porras, Isabel (coordinadores): De la responsabilidad individual a la culpabilización de la víctima. El papel del paciente en la prevención de la enfermedad (Barras)

Müller, Christian: De l'asile au centre psychosocial. Esquisse d'une histoire de la psychiatrie suisse (Modestin)

Müller, Gerhard H. (Hrsg.): Themen zur Geschichte der Biologie. Zum 60. Geburtstag von Professor Dr. Armin Geus (Höxtermann) . . . . . . . . . . . . . . . . . . . .

Navarro Brotóns, Victor; Galdeano, Enrique Rodriguez: Matemáticas, cosmología y humanismo en la España del siglo XVI. Los Comentarios al segundo libro de la Historia Natural de Plinio de Jerónimo Muñoz (Gantenbein) $\ldots \ldots \ldots \ldots \ldots \ldots \ldots \ldots \ldots \ldots \ldots$

Osten, Sigrid von: Das Alchemistenlaboratorium von Oberstockstall (Gantenbein) .... 280 280

Pastore, Alessandro: Il medico in tribunale. La perizia medica nella procedura penale

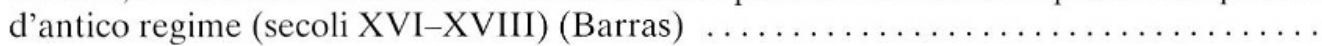

Pelling, Margaret (ed.): The common lot. Sickness, medical occupations and the urban poor in early modern England (Steinbrecher)

Pictet, Marc-Auguste: Correspondance: sciences et techniques. Tome I: Les Correspondants genevois. Tome II: Les Correspondants français (Kanz) ..................

Pietikäinen, Petteri: C. G. Jung and the psychology of symbolic forms (Kast) ........

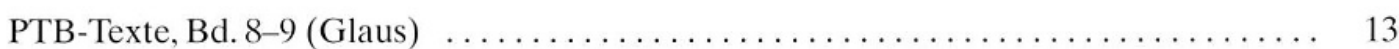

Rashed, Roshdi; Vahabzadeh, B.: Al-Khayyam mathématicien (Ineichen) . . . . . . . . 284

Schiebinger, Londa: Am Busen der Natur. Erkenntnis und Geschlecht in den Anfängen der Wissenschaft (Sauerteig) . . . . . . . . . . . . . . . . . . . . . . . . . .

Schiferer, H. Ruediger: Alfred Adler. Eine Bildbiographie (Fischer-Homberger) . . .... 286 138

Schlumbohm, Jürgen; Duden, Barbara; Gélis, Jacques; Veit, Patrice (Hrsg.): Rituale der Geburt. Eine Kulturgeschichte (Borkowsky) $\ldots \ldots \ldots \ldots \ldots \ldots \ldots \ldots \ldots \ldots \ldots \ldots \ldots$

Schmutz, Hans-Konrad (Hrsg.): Phantastische Lebensräume, Phantome und Phantasmen

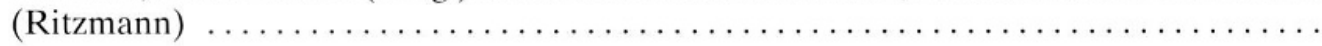


Schnalke, Thomas; Wiesemann, Claudia (Hrsg.): Die Grenzen des Anderen. Medizingeschichte aus postmoderner Perspektive (Ritzmann)

Schüle, Rose-Claire: L'inventaire lexicologique du parler de Nendaz (Valais). Vol. 2:

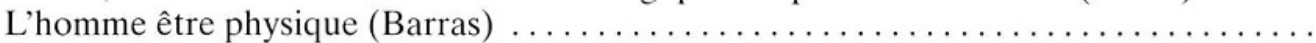

Schulte am Esch, Jochen; Goerig, Michael (eds): Anaesthetic equipment in the history

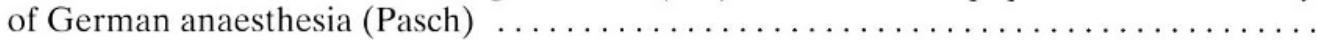

Schulte am Esch, Jochen; Goerig, Michael (eds): The Fourth International Symposium on the History of Anaesthesia (Hamburg, April 1997) (Pasch)

Segala, Marco: I fantasmi, il cervello, l'anima: Schopenhauer, l'occulto e la scienza (Kanz)

Seiler, Eberhard (ed.): The role of metrology in economic and social development

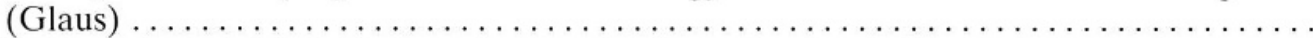

Sigrist, René; Barras, Vincent; Radcliff, Marc: Louis Jurine, chirurgien et naturaliste

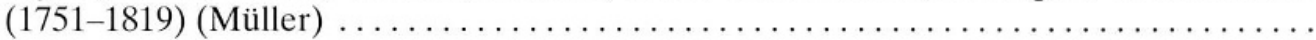

Sonntag, Otto (ed.): John Pringle's correspondence with Albrecht von Haller (Boschung) 288

Soukup, Rudolph Werner; Mayer, Helmut: Alchemistisches Gold, Paracelsistische Pharmaka. Laboratoriumstechnik im 16. Jahrhundert (Gantenbein)

Spallanzani, Lazzaro: Edizione nazionale delle opere di Lazzaro Spallanzani. Parte quarta: Opere edite direttamente dall'autore (volume terzo, 1776-1779, Secondo

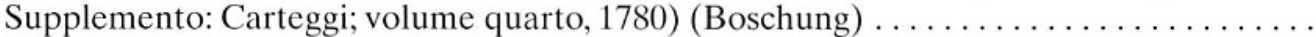

Stautz, Burkhard: Untersuchungen von mathematisch-astronomischen Darstellungen auf mittelalterlichen Astrolabien islamischer und europäischer Herkunft (Gantenbein)

Steinke, Hubert (Hrsg.): Der nützliche Brief. Die Korrespondenz zwischen Albrecht von Haller und Christoph Jakob Trew 1733-1763 (Kanz) . . . . . . . . . . . . . . . . .

Stelzner, Friedrich: Lebenswellen, Lebenswogen eines Chirurgen (Naef) . . . . . . . . .

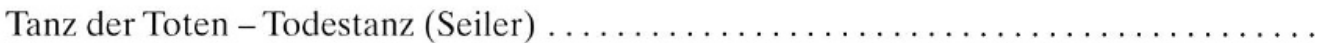

Trudeau, Richard: Die geometrische Revolution (Ineichen)

Uebele, Susanne: Bauten der Max-Planck-Gesellschaft zur Förderung der Wissen-

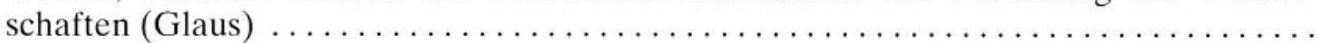

Veröffentlichungen aus dem Archiv zur Geschichte der Max-Planck-Gesellschaft

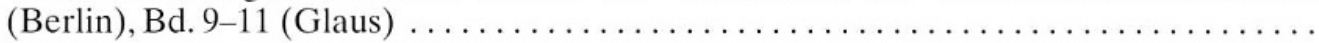

Hugo de Vries 1848-1998 (Dalang)

Wagner-Egelhaaf, Martina: Die Melancholie der Literatur. Diskursgeschichte und

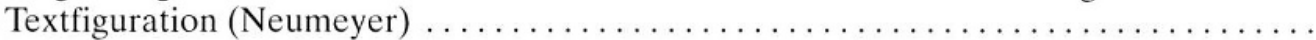

Weber, Kaspar: «Es geht ein mächtiges Sehnen durch unsere Zeit». Reformbestrebungen der Jahrhundertwende und Rezeption der Psychoanalyse am Beispiel der Biografie von Ernst Schneider 1878-1957 (Stückelberger) 
Wegeleben, Christel: Beständeübersicht des Archivs zur Geschichte der Max-Planck-

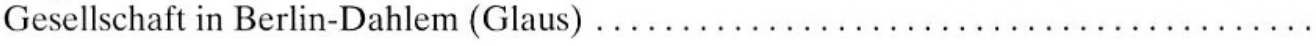

Weiner, Dora B.: Comprendre et soigner. Philippe Pinel (1745-1826), la médecine et

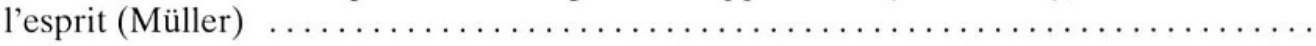

Wiemers, Kurt: Weiter atmen - leben! Wege und Umwege zur Anästhesie und Intensiv-

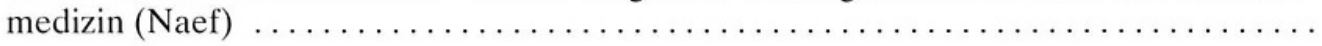

